\title{
1 Biobanken in der öffentlichen Wahrnehmung: Verständnis, Interesse und Motivation von Probenspendern in Deutschland
}

Wiebke Lesch, Antje Schütt und Roland Jahns

- Biobanken entwickeln sich zu (multi-)nationalen Plattformen für die biomedizinische Forschung, aber die Öffentlichkeit bekommt davon nur wenig mit.

- Trotz Aufklärung weiß die Mehrheit der Spender nicht, was eine Biobank ist und wofür ihre Proben verwendet werden.

- Die Grundeinstellung gegenüber Wissenschaft und Forschung ist jedoch überwiegend positiv, und die Motive für eine Spende sind altruistischer Natur.

- Die Einführung des „Broad Consent“ erfordert ein Umdenken in der Spenderbeziehung: Biobanken müssen einen offenen Dialog mit Spendern führen, bei der Transparenz und Vertrauensbildung an erster Stelle stehen.

Biobanken boomen. Sie lagern weder Geld noch Gold, sondern Gewebe, Blut oder andere Körperflüssigkeiten von Menschen für die Forschung. Die Zahl der Biobanken ist in den letzten Jahren weltweit rasant gewachsen, denn sie sind von enormer Bedeutung für die biomedizinische Forschung. Mehr als 5oo Biobanken mit vielen Millionen Proben gibt es laut BBMRI ${ }^{1}$ allein in Europa - Ten-

1 Biobanking and Biomolecular Resources Research Infrastructure, eine der ersten, von der Europäischen Kommission geförderten Europäischen Forschungsinfrastrukturen, ist heute als Biobanking and BioMolecular resources Research Infrastructure - European Research Infrastructure Consortium (BBMRI-ERIC) aktiv mit dem Ziel, eine europaweite Forschungsinfrastruktur von Biobanken und biomolekularen Ressourcen zu etablieren, zu betreiben und weiterzuentwickeln. Der German Biobank Node ist der nationale Knoten von BBMRI-ERIC in Deutschland. 
denz steigend. Doch wofür werden diese Proben gebraucht? Komplexe Krankheiten wie Krebs, Diabetes oder Autoimmunerkrankungen haben komplexe Ursachen und Mechanismen. Biobanken unterstützen die Forschung, indem sie dazu beitragen, diese krankheitsbedingten Veränderungen aufzuklären. Sie sind unverzichtbar für die biomedizinische Forschung und damit auch für die Entwicklung und Überprüfung neuer Behandlungsansätze. BBMRI beschreibt Biobanken deshalb als „... a new type of large-scale research infrastructure located at the intersection of biomedical research and information technology, at the border between research and biomedicine“ (BBMRI 2013).

In den letzten Jahren wurden in Deutschland erstmals große zentralisierte Biobanken an mehreren Universitätskliniken errichtet, die langfristig viele Millionen Proben von Patienten mit einer großen Bandbreite an Krankheiten einlagern werden. Diese Aktivitäten werden durch den Aufbau eines deutschen Biobanken-Knotens (German Biobank Node, GBN) bzw. die German Biobank Alliance ( $\mathrm{GBA}$ ) als zentrale Kontakt- und Vermittlungsstelle für Biobanken koordiniert und ergänzt ${ }^{2}$. In der Öffentlichkeit sind Biobanken so gut wie unbekannt: weder mit dem Begriff noch mit der Tätigkeit einer Biobank können Laien etwas anfangen (Gaskell 2010, 2011). Aufgrund ihrer wachsenden Bedeutung für die medizinische Forschung fordern Experten eine stärkere Sichtbarkeit und Einbindung von Öffentlichkeit und Probenspendern (Deutscher Ethikrat 2010): Sinn, Zweck und Nutzen von Biobanken müssen der Öffentlichkeit transparent und nachvollziehbar vermittelt werden.

\section{Befragung von Biomaterialspendern in Deutschland}

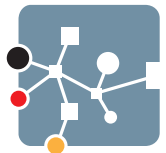

German

Biobank Node

bbmri.de

Der German Biobank Node (GBN) hat gemeinsam mit einigen zentralisierten Biobanken deutscher Universitätsklinika eine Spenderbefragung initiiert, um die Bekanntheit und die Einstellung von Patienten gegenüber Biobanken zu untersuchen. Zwischen Juli 2014 und Juni 2015 wurden an den Universitätskliniken Hannover, Heidelberg und Würzburg Fragebögen an Patienten und Probanden verteilt. Ziel war es, Einstellungen, Motivationen und Verständnis gegenüber dem Biobanking in Deutschland zu ermitteln. Die Ergebnisse geben wichtige Hinweise darauf, wie Biobanken ihr Stakeholder Involvement gestalten können.

Alle 187 befragten Spender hatten zuvor einer Biomaterialspende im Rahmen ihrer Behandlung an der Klinik oder im Rahmen einer klinischen Studie zugestimmt und wurden über die Spende schriftlich und mündlich aufgeklärt. 60\% der befragten Männer und Frauen waren Patienten mit jeweils unterschiedlichen Erkrankungen, 40\% waren gesunde Probanden.

2 Siehe: http://www.gesundheitsforschungbmbf.de/de/2638.php bzw. http://www.gesundheitsforschung-bmbf.de/ $\mathrm{de} / 5793$ 
1 Biobanken in der öffentlichen Wahrnehmung:

Kernthemen der Befragung umfassten:

- das Verständnis darüber, was eine Biobank ist,

- das Verständnis darüber, wozu die gespendeten Biomaterialien und die gesammelten Daten verwendet werden,

- die Einstellung und Motivation gegenüber einer Biomaterialspende,

- das Interesse an Forschungsaktivitäten und -ergebnissen.

\subsection{Was verstehen Spender unter einer Biobank?}

Die zahlenmäßig größte Erhebung zur Bekanntheit von Biobanken, der „Eurobarometer Survey: Life Sciences and Biotechnology“, kam 2010 zu dem Ergebnis, dass $67 \%$ der Europäer noch nie von einer Biobank gehört haben. Der geringe Bekanntheitsgrad in der allgemeinen Bevölkerung würde sich auch unmittelbar auf die Bereitschaft zur Teilnahme an einer Biobank niederschlagen, so die Autoren der Studie. Nur 12\% der deutschen Bevölkerung können sich laut Eurobarometer vorstellen, ihr Biomaterial einer Biobank zur Verfügung zu stellen, und jeder zweite Deutsche hat Bedenken bei der Vorstellung, dass sein Biomaterial in einer Biobank eingelagert wird.

Um besser zu verstehen, welche Vorstellungen Patienten und Probanden von Biobanken haben, hat der German Biobank Node (GBN) eine Befragung gezielt unter Biomaterialspendern durchgeführt. Da die von GBN befragten Patienten und Probanden bereits eingewilligt hatten, Biomaterial zu spenden, wollten wir zunächst ihr Wissen über die Biomaterialspende abfragen. Auf die Frage: „Bitte beschreiben Sie mit eigenen Worten ganz kurz, was Sie sich unter einer Biobank vorstellen." konnten 32\% der Patienten und Probanden korrekt beschreiben, was eine Biobank ist. Immerhin 68\% der Biomaterialspender hatten dagegen trotz vorheriger Aufklärung durch medizinisches Fachpersonal keine oder nur eine ungenaue Vorstellung von einer Biobank. In der untersuchten Spenderstichprobe war der Kenntnisstand über Biobanken somit sehr gering.

\subsection{Wofür werden die gespendeten Materialien verwendet?}

Der „Informed Consent“ ist eine rechtlich vorgeschriebene Verfahrensweise, die sicherstellen soll, dass ein Spender (Patient oder Studienteilnehmer) die Risiken und den möglichen Nutzen einer Biomaterialspende versteht und dann freiwillig eine „informierte Entscheidung“ trifft. Der Spender wird deshalb vor jeder Spende von qualifiziertem medizinischem Fachpersonal mündlich über die Verwendung seiner Bioproben aufgeklärt, zusätzlich erhält er eine Informationsschrift und unterzeichnet eine Einwilligungserklärung. Von den vermittelten Informationen kommt leider tatsächlich nicht viel beim Spender an: nur jeder zweite Patient (58\%) kann sich in der GBNBefragung daran erinnern, über die Verwendung seiner Daten und Proben in der Biobank aufgeklärt worden zu sein (s. Abb. 1). Nur zwei Drittel der Be- 
Wurden Sie über die Verwendung Ihrer Daten und/oder Ihrer Biomaterialien in der Biobank aufgeklärt? $(n=187)$

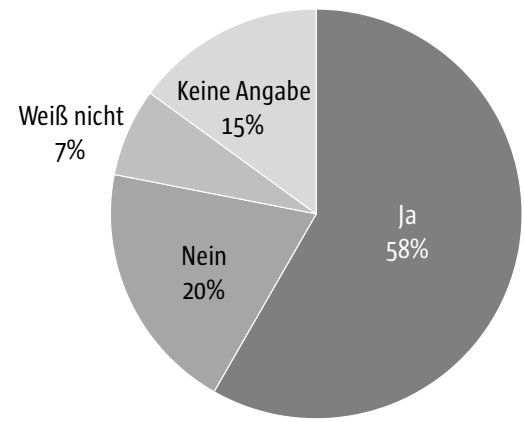

Abb. 1 Verwendung der Daten und Proben

fragten erinnern sich daran, eine Einwilligungserklärung unterschrieben zu haben. Alter ist ein signifikanter Einflussfaktor: je jünger die Spender, desto besser erinnern sie sich an die Inhalte und den Prozess der Aufklärung.

Weiterhin wurde in einer offenen Frage abgefragt, an welche für sie bedeutsamen Inhalte aus der Einwilligungserklärung sich die Befragten erinnern. $67 \%$ der Studienteilnehmer erinnern sich an keine spezifischen Inhalte aus der Aufklärung. 33\% der Befragten nennen folgende Punkte (mit abnehmender Häufigkeit der Nennung):

- Forschungszweck

- Datenschutz

- Widerruf und Freiwilligkeit der Einwilligung

- Datenspeicherung

- Lagerung der Proben

- Datenweitergabe

Für die insgesamt sehr geringe Aufnahme von Informationen gibt es verschiedene Erklärungsmöglichkeiten. Aus diversen Studien ist bekannt, dass das Ergebnis eines Aufklärungsgespräches von vielen Details abhängt: von der Sprache, die in der Einwilligungserklärung verwendet wird, vom Ablauf des Aufklärungsprozesses, bis hin zur Teilnahmemotivation und dem unterschiedlich ausgeprägten Interesse eines Teilnehmers an Informationen. Umso interessanter ist es, sich näher damit auseinanderzusetzen, was Teilnehmer dazu motiviert, Biomaterial zu spenden.

\subsection{Einstellung zu und Motive für eine Biomaterialspende}

Die öffentliche Meinung zu Wissenschaft und Forschung und auch zu Biobanken ist überwiegend positiv (BBMRI 2013). Das spiegelt sich auch in der 
1 Biobanken in der öffentlichen Wahrnehmung:

Verständnis, Interesse und Motivation von Probenspendern in Deutschland

Gefühl bei Spende: Positiv versus negativ $(n=187)$

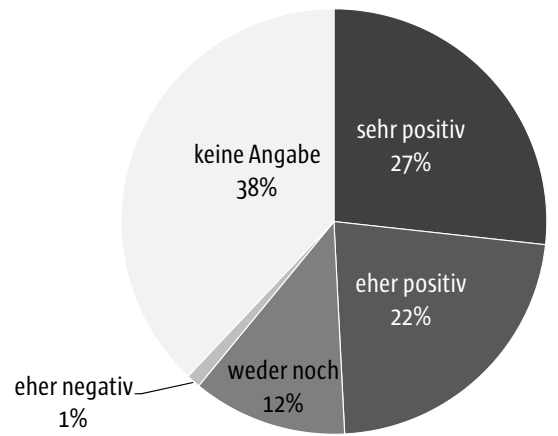

Abb. 2 Einstellung zur Biomaterialspende

GBN-Patientenbefragung wider. Jeder zweite Spender (49\%) ist einer Biomaterialspende gegenüber positiv eingestellt (s. Abb. 2) und würde wieder Biomaterial spenden $(80 \%)$.

Dennoch ist die Teilnahme an einer Biobank auch an Erwartungen seitens der Spender geknüpft. Es bestehen durchaus Bedenken hinsichtlich des Schutzes der Persönlichkeitsrechte (Privacy) und des Datenschutzes (Data protection).

Doch was bewegt Probenspender letztlich dazu, ihr Biomaterial und die zugehörigen Daten für Forschungszwecke zur Verfügung zu stellen? Die Motive für eine Spende sind überwiegend altruistischer Natur: Patienten wollen „Forschung und Wissenschaft unterstützen“ und „zukünftigen Patientengenerationen helfen“ (s. Abb. 3). Die Mehrheit der Patienten versteht, dass sie persönlich keinen unmittelbaren Eigennutzen von ihrer Spende haben, die Biomaterialspende aber anderen Menschen helfen könnte.

Warum haben Sie sich dafür entschieden, Blut, Gewebe, Urin oder Ähnliches für die Biobank zur Verfügung zu stellen? (Offene Frage, Mehrantworten möglich) $(n=187)$

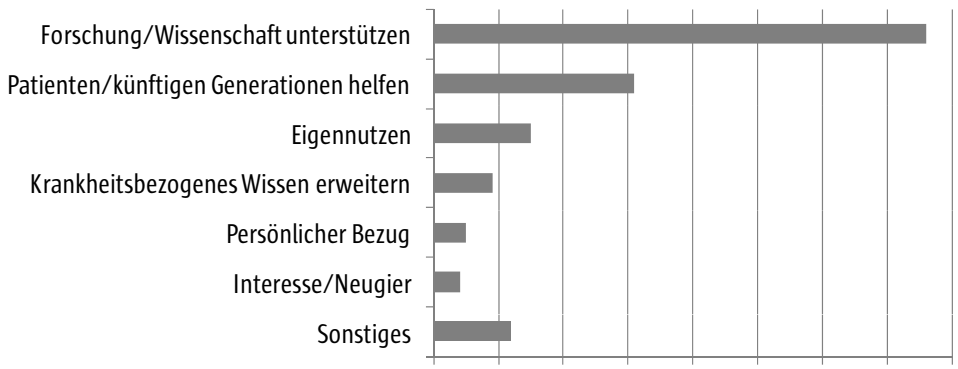


Andere Studien zu diesem Thema führen neben diesen beiden Kernmotiven zudem die Beziehung zum Arzt, das Vertrauen in die durchführende Institution (Nobile 2016, Lidz 2006) sowie das generelle Vertrauen in die Gesellschaft (Gaskell 2011) als Einflussfaktoren auf die Bereitschaft zur Biomaterialspende an. Insgesamt kann man festhalten, dass die Motivation, sich an einer Studie zu beteiligen, durch ein komplexes Zusammenspiel von persönlichen und gesellschaftlichen Faktoren geprägt ist, in welchem Vertrauen das Kernmotiv für die Biomaterialspende ist.

Die Befragung zeigt ebenfalls, dass eine hohe Bereitschaft für den in der Forschung angestrebten „Broad Consent“ vorhanden ist. So gaben $61 \%$ der Befragten an, dass ihre Probe jederzeit für verschiedene Forschungsprojekte eingesetzt werden darf (s. Abb. 4).

Von BBMRI durchgeführte Studien mit Fokusgruppen weisen darauf hin, dass der von modernen Biobanken angestrebte „Broad Consent“ (die Einwilligung zur Nutzung der Biomaterialspende ohne festgelegten Forschungszweck und über den Tod hinaus) jedoch einen hohen Grad an Transparenz, Offenheit über die Ziele und ein Grundvertrauen in die Institution erfordert (BBMRI 2013). Die Beziehung zum Spender sollte nicht als einmalige Interaktion betrachtet, sondern als langfristige Beziehung verstanden werden, die von einer Kultur des „Gebens und Nehmens“ zwischen Biomaterialspender und Biobank geprägt ist, so die Autoren.

\section{Was denken Biomaterialspender?}

Brigitte ist ein fiktiver Charakter, eine sogenannte Persona. Sie steht repräsentativ für die Stakeholdergruppe der Biomaterialspender und bildet prototypisch die Ziele, Wünsche und Bedürfnisse dieser Gruppe ab. Die Beschreibung der Persona ist das Ergebnis von qualitativen und quantitativen Nutzerbefragungen.

Welcher dieser Aussagen stimmen Sie zu? (Mehrfachantworten möglich)

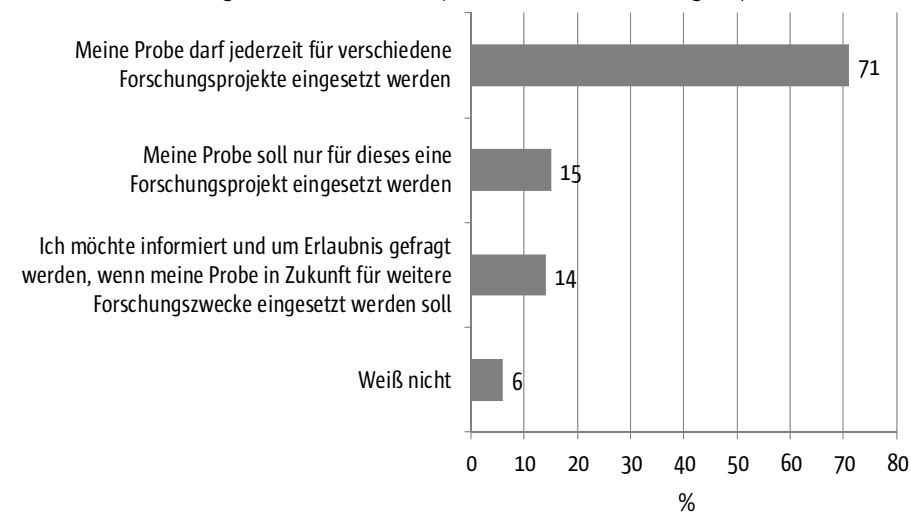

Abb. 4 Einstellung der Spender zum Broad Consent 


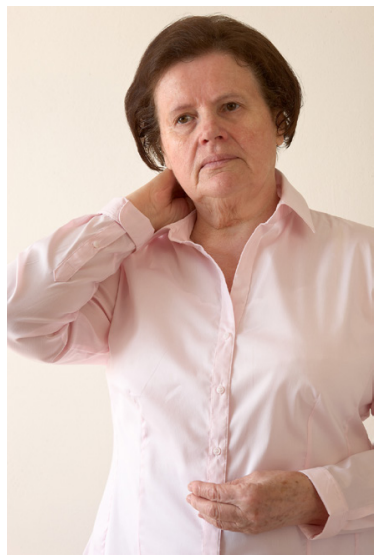

Abb. 5 Brigitte - Persona eines Biomaterialspenders (Copyright: Edyta Guhl/Fotolia)

Bei Brigitte wurde kürzlich Brustkrebs festgestellt. Von ihrem niedergelassenen Arzt wird sie zur Behandlung in die Uniklinik geschickt. Die Operation steht unmittelbar bevor und sie ist nervös und ängstlich, wie diese verlaufen wird. Im Zuge der OP-Vorbereitung wird sie von ihrer behandelnden Ärztin angesprochen, ob sie einen Teil des bei der Operation entnommenen Gewebes für die Forschung spenden würde. Sie hat generell nichts dagegen, interessiert sich aber in diesem Moment mehr für ihre persönliche Gesundheit als für dieses Thema. Von Biobanking hat sie noch nie etwas gehört, vertraut aber ihrem Arzt, dass alles rechtens ist. Sie hofft, dass ihre Spende wenigstens zukünftigen Generationen etwas nützt. Zu einem späteren Zeitpunkt interessiert sie sich dafür, welchen Beitrag sie mit ihrer Probe leisten konnte, und würde gerne erfahren, welche Ergebnisse aus der Forschungsarbeit herausgekommen sind.

Gründe eines Spenders für eine Biomaterialspende:

1. Gutes tun

2. Wissenschaft und Forschung unterstützen

Gründe, die einen Spender potenziell von einer Spende abhalten:

1. mangelnde Bekanntheit bzw. Vertrautheit mit dem Thema

2. vorhandene Bedenken hinsichtlich des Schutzes der Privatsphäre oder der Einwilligung

Was der Spender wissen möchte:

- Was muss ich tun?

- Wieviel Zeit muss ich aufbringen?

- Was wird mit meiner Probe gemacht?

- Wer ist verantwortlich?

- Was sind die Teilnahmebedingungen?

- Wird meine Probe kommerziell genutzt?

- Kann meine Probe mit meiner Person in Verbindung gebracht werden?

- Wie sicher sind meine Daten?

Quellen: GBN Nutzerbefragung, UKCRC Tissue Directory and Coordination Centre (2016) 


\subsection{Interesse an Forschungsaktivitäten und -ergebnissen}

Die Spender sind ohne Zweifel eine der zentralen Stakeholdergruppen im Biobanking. Ohne die Bereitschaft der Menschen, Biomaterial zu spenden, kann es nur schwerlich medizinische Forschung geben. Bedingt durch das zunehmende Bedürfnis moderner Biobanken nach einem „Broad Consent“ wird man auch das Verhältnis zwischen Biobanken und Biomaterialspendern überdenken müssen (Strech et al. 2016). Es wird sich langsam von einem obligatorischen „Einholen“ einer Einwilligung hin zu einem Modell entwickeln, in dem die Biomaterialspender eine aktivere Rolle spielen werden. Vor diesem Hintergrund haben wir deutsche Biomaterialspender gefragt, wie stark ihr Interesse an den mit ihren Proben durchgeführten Studien und Forschungsergebnissen ist (s. Abb. 6). Die Ergebnisse zeigen eindeutig, dass ein hohes Interesse besteht: Drei von vier Patienten (73\%) wünschen sich eine Rückmeldung zu den mit Hilfe der Biomaterialspenden erzielten Forschungsergebnissen.

Auch bei unerwarteten Zusatzbefunden wünscht die Mehrheit der Patienten (70\%) eine individuelle Rückmeldung (s. Abb. 7). Jeder zweite Patient (50\%) wünscht eine Rückmeldung, wenn ein erhöhtes Risiko für eine Erkrankung festgestellt wird, weitere 20\% nur dann, wenn eine Möglichkeit besteht, dieses Risiko zu beeinflussen.

Biobanken sollten das hohe Informationsbedürfnis der Patienten und Probanden bezüglich der von den Forschern erzielten Forschungsergebnisse und die Art und Weise der Rückmeldung von Zusatzbefunden in ihre Informationspolitik bzw. Statuten integrieren. Falls dem Wunsch nach einer individuellen Rückmeldung bei Zusatzbefunden nicht entsprochen werden kann, muss das klar kommuniziert und begründet werden (Erwartungsmanagement). Eine allgemein verständliche Darstellung von Forschungsergeb-

Würden Sie gern erfahren, was die Wissenschaftler damit untersuchen bzw. herausfinden? $(n=187)$

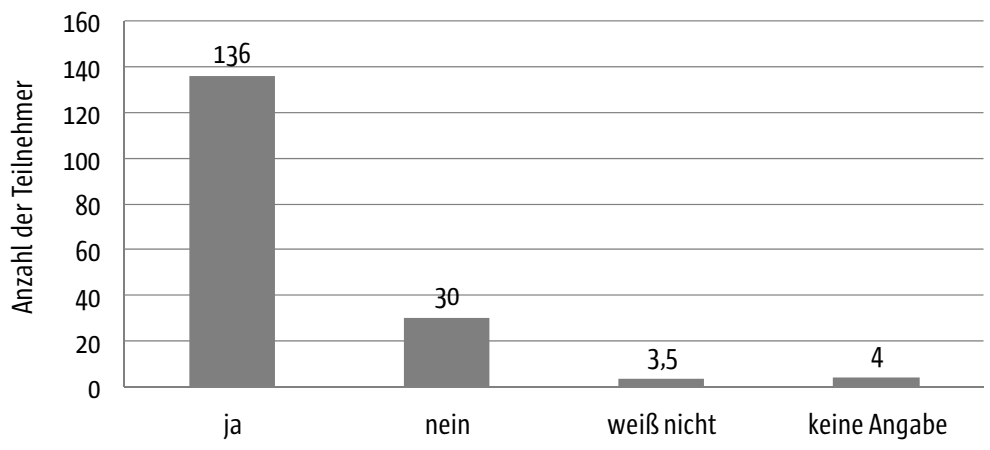

Abb. 6 Interesse an Forschungsergebnissen 
1 Biobanken in der öffentlichen Wahrnehmung:

Verständnis, Interesse und Motivation von Probenspendern in Deutschland

Falls ein Forscher bei der Untersuchung Ihrer Probe ein erhöhtes Risiko für eine bestimmte Erkrankung feststellt, würden Sie darüber informiert werden wollen? $(n=187)$

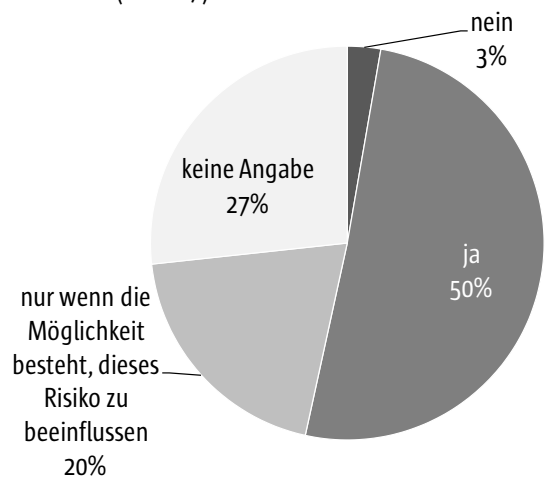

Abb. 7 Wunsch der Spender nach einer persönlichen Rückmeldung

nissen wird zukünftig zum Rückmeldungsstandard gehören, wenn man die Erwartungen der Biomaterialspender nicht enttäuschen möchte. „People need to feel that they are part of something larger and that their donation feeds into a mutual, respectful relationsship. They want to be appreciated as donors and be treated well“", fassen Gottweis et al. die derzeitige Entwicklung zusammen (Gottweis 2011). Biobanken werden nicht mehr nur die Interessen der Forscher vertreten können, sondern müssen sich zunehmend auch als Mediatoren zwischen Forschern, Biomaterialspendern und weiteren Stakeholdergruppen verstehen.

\subsection{Empfehlungen für die Biobankenkommunikation}

Biobanken befinden sich in einem tiefgreifenden Transformationsprozess: die einst übersichtlichen lokalen Sammlungen mit festgelegtem Verwendungszweck für die Proben entwickeln sich zu (multi-)nationalen Plattformen für die biomedizinische Forschung. Die allgemeine Öffentlichkeit und die Biomaterialspender bekommen davon bisher wenig mit. Biobanken brauchen aber die Unterstützung von Patienten und Probanden, die bereit sind, ihre Biomaterialien und Daten für die Forschung bereitzustellen und natürlich auch das Vertrauen der Öffentlichkeit. Deshalb sind die Wünsche und Bedürfnisse von Biomaterialspendern nicht länger zu vernachlässigen. Ein Verlust des Vertrauens würde sich nicht nur negativ auf die Spendebereitschaft auswirken, sondern dem Ruf der gesamten Biobankbranche schaden. Deshalb ist es für Biobanken unausweichlich, sich proaktiv in den öffentlichen Dialog einzubringen. 


\section{Empfehlungen}

Basierend auf einer vom German Biobank Node (GBN) durchgeführten Befragung, haben wir zusammenfassend folgende Empfehlungen für die Interaktion der Biobank mit Spendern entwickelt:

- Angesichts der zunehmenden Zentralisierung der Biobankstrukturen, die mit einer Abnahme der direkten persönlichen Beziehung zwischen Forscher und Biobankspender einhergeht, ist es notwendig, die Spender (aber auch alle anderen Stakeholder) stärker einzubinden. Die Biobank muss einen offenen Dialog mit allen Stakeholdergruppen führen.

- Behalten Sie die Bedürfnisse und Erwartungen von Patienten und Probanden im Blick und gehen Sie auf deren Erwartungen ein. Biomaterialspender müssen über die Ziele, Prozesse und Forschungsergebnisse transparent, zielgruppengerecht und zu einem geeigneten Zeitpunkt aufgeklärt werden.

- Vermitteln und danken Sie dem Biomaterialspender, dass er ein Teil des auf die Zukunft gerichteten medizinischen Wissenschaftssystems ist und mit seiner Spende zum Fortschritt der medizinischen Forschung beiträgt. Ein positives Signal im Hinblick auf die Spende verstärkt die positive Einstellung des Biomaterialspenders gegenüber Wissenschaft und Forschung und führt letztlich zu einer höheren Beteiligung.

- Gehen Sie verantwortungsvoll mit dem Vertrauen der Spender um. Binden Sie Spender, Betroffenengruppen und/oder Vertreter der Öffentlichkeit in die Governancestrukturen der Biobank ein, um Vertrauen gegenüber Biobanken auf- und auszubauen.

- Betrachten Sie die Interaktion mit dem Spender nicht als ein einmaliges Ereignis, das mit der Spende abgeschlossen ist, sondern als ein gegenseitiges „Geben und Nehmen“. Pflegen Sie, soweit möglich, die Beziehung zum Biomaterialspender.

- Vertreten Sie als Biobankbetreiber nicht nur die Interessen der wissenschaftlichen Community, sondern auch die Interessen der Spender. Die Biobank sollte als Vermittler zwischen den Interessen der verschiedenen Stakeholdergruppen auftreten. Biomaterialspender sind dabei ein entscheidender Teil des Systems.

Jede Biobank sollte eine zu ihren Zielen und ihrem Kontext passende Partizipationsstrategie entwickeln. Das Ausmaß der Einbindung der Stakeholder kann dabei stark variieren: von einer eher beratenden oder ideengebenden bis hin zu einer Entscheidungsfunktion ist alles möglich bzw. denkbar.

Beispielhaft seien in Tabelle 1 einige Partizipationsformen mit ihren Möglichkeiten und Grenzen aufgeführt. 
1 Biobanken in der öffentlichen Wahrnehmung:

Verständnis, Interesse und Motivation von Probenspendern in Deutschland

Tab. 1 Partizipationsformen (aus Boeckhout et al. 2014, mit freundlicher Genehmigung).

\begin{tabular}{|c|c|c|c|c|c|}
\hline $\begin{array}{l}\text { Form der } \\
\text { Partizipation }\end{array}$ & Passend für & $\begin{array}{l}\text { Level der } \\
\text { Partizipation }\end{array}$ & Varianten & $\begin{array}{c}\text { Grad der } \\
\text { Unterstützung }\end{array}$ & $\begin{array}{l}\text { Spezifische } \\
\text { Themen }\end{array}$ \\
\hline $\begin{array}{l}\text { Einbindung } \\
\text { von Patienten- } \\
\text { organisation im } \\
\text { Management }\end{array}$ & $\begin{array}{l}\text { Forschung in Gebieten } \\
\text { mit aktiven Patienten- } \\
\text { organisationen }\end{array}$ & $\begin{array}{l}\text { Einbindung in } \\
\text { Entscheidungen }\end{array}$ & $\begin{array}{l}\text { Direkte Ein- } \\
\text { bindung oder } \\
\text { indirekte Monito- } \\
\text { ringfunktion }\end{array}$ & limitiert & $\begin{array}{c}\text { Vereinbarung } \\
\text { über das Ausmaß } \\
\text { der Einbindung }\end{array}$ \\
\hline Advisory Board & $\begin{array}{c}\text { Biobanken mit } \\
\text { strategischen und } \\
\text { praktischen Fragen } \\
\text { bezüglich der Spender- } \\
\text { perspektive }\end{array}$ & $\begin{array}{l}\text { Aussprache von } \\
\text { Empfehlungen }\end{array}$ & $\begin{array}{c}\text { Biomaterial- } \\
\text { spender oder Pa- } \\
\text { tienten Advisory } \\
\text { Board }\end{array}$ & $\begin{array}{c}\text { Bedarf } \\
\text { erheblicher } \\
\text { Unterstützung }\end{array}$ & $\begin{array}{l}\text { Organisation } \\
\text { verpflichtet sich, } \\
\text { ein Mandat } \\
\text { einzuräumen }\end{array}$ \\
\hline $\begin{array}{l}\text { Direkte } \\
\text { Einflussnahme }\end{array}$ & $\begin{array}{c}\text { Tissue Biobanken } \\
\text { und institutionelle } \\
\text { Biobanken }\end{array}$ & $\begin{array}{l}\text { Beratung, } \\
\text { Beisteuerung } \\
\text { von Ideen, in- } \\
\text { direkte Rolle bei } \\
\text { Entscheidungen }\end{array}$ & $\begin{array}{c}\text { Patienten } \\
\text { Advisory Board }\end{array}$ & limitiert & $\begin{array}{c}\text { Hintergrundwis- } \\
\text { sen der Advisory } \\
\text { Board Mitglieder } \\
\text { notwendig }\end{array}$ \\
\hline Patientenwissen & $\begin{array}{l}\text { Klinische Biobanken } \\
\text { und Biobankforschung }\end{array}$ & $\begin{array}{l}\text { Kooperationen, } \\
\text { Beisteuerung } \\
\text { von Ideen, } \\
\text { Konsultationen }\end{array}$ & $\begin{array}{l}\text { Beitrag zur Strate- } \\
\text { gie, Involvement } \\
\text { in die Organisa- } \\
\text { tionsentwicklung, } \\
\text { Beratung in der } \\
\text { Methodologie }\end{array}$ & variiert & $\begin{array}{l}\text { Training von } \\
\text { Patientenexper- } \\
\text { ten, Bereitschaft } \\
\text { zum Dialog }\end{array}$ \\
\hline Fokusgruppen & $\begin{array}{l}\text { Biobanken und } \\
\text { Forschung, die eine } \\
\text { systematische Explo- } \\
\text { ration der öffentlichen } \\
\text { Meinung benötigen }\end{array}$ & $\begin{array}{l}\text { Konsultationen, } \\
\text { Beisteuerung von } \\
\text { Ideen }\end{array}$ & $\begin{array}{l}\text { Gruppeninter- } \\
\text { views, metho- } \\
\text { disch fundierte } \\
\text { Befragungen }\end{array}$ & variiert & $\begin{array}{l}\text { Zeitplanung, } \\
\text { Budget, } \\
\text { Organisation }\end{array}$ \\
\hline $\begin{array}{l}\text { Konsultations- } \\
\text { runden }\end{array}$ & $\begin{array}{l}\text { Zum Review oder } \\
\text { Feedback zu spezi- } \\
\text { fischen Vorhaben }\end{array}$ & Konsultation & $\begin{array}{l}\text { Limitiert (z.B. } \\
\text { Fokusgruppen, } \\
\text { breite Befragungen) }\end{array}$ & variiert & $\begin{array}{l}\text { Zeitplanung, } \\
\text { Budget, } \\
\text { Organisation }\end{array}$ \\
\hline $\begin{array}{l}\text { Online } \\
\text { Engagement }\end{array}$ & $\begin{array}{l}\text { Onlinevarianten von } \\
\text { allen oben genannten } \\
\text { Formen }\end{array}$ & $\begin{array}{l}\text { Konsultationen, } \\
\text { Beisteuerung von } \\
\text { Ideen }\end{array}$ & siehe oben & variiert & $\begin{array}{l}\text { Zeitplanung, } \\
\text { Budget, } \\
\text { Organisation }\end{array}$ \\
\hline
\end{tabular}

\section{Das Projekt wurde vom Bundesministerium für Bildung und Forschung im Rahmen des German Biobank Node unter dem Förderkennzeichen 01EY1301 gefördert.}

\section{Quellenangaben}

Boeckhout M, Reuzel R, Zielhuis $G$ (2014) The donor as partner. How to involve patients and the public in the governance of biobanks and registries. BBMRI-NL, November 2014. http://www.bbmri.nl/on_offer/donoras-partner/

Biobanking and Biomolecular resources Research Infrastructure (BBMRI) (2013) Biobanks and the public - Governing Biomedical Research Ressources in Europe

Deutscher Ethikrat (2010) Humanbiobanken für die Forschung. Stellungnahme.

Gaskell G. eds. (2010) Europeans and Biotechnology in 2010. Winds of Change? Eurobarometer. European Union.

Gaskell G, Gottweis H (2011) Biobanks need publicity. Nature 471:159-171 
Gottweis H, Gaskell G, Starkbaum I (2011) Connecting the public with biobank research: reciprocity matters. Nature Reviews Genetics 12, 738-739

Nobile H, Bergmann M, Moldenhauer I, Borry P (2016) Participants' Accounts on Their Decision to Join a Cohort Study With an Attached Biobank: A Qualitative Content Analysis Study Within Two German Studies. J Empir Res Hum Res Ethics. 2016 Jul;11(3):237-49.

Lidz CW (2006) The therapeutic misconception and our models of competency and informed consent. Behav Sci Law. 2006;24(4):535-46.

Strech D, Bein S, Brumhard M, Eisenmenger W, Glinicke C, Herbst T, Jahns R, von Kielmansegg S, Schmidt G, Taupitz I, Tröger HD (2016) A template for broad consent in biobank research. Results and explanation of an evidence and consensus-based development process. Eur | Hum Genetics 2016 Jun;59(6-7):295-309.

UKCRC Tissue Directory and Coordination Centre (2016) Personas. https://www.biobankinguk.org/personas/ (20. September 2016) 